\title{
Mobile Health for Traumatic Brain Injury: A Systematic Review of the Literature and Mobile Application Market
}

\author{
Edward Christopher ${ }^{1}$, Kareem W. Alsaffarini ${ }^{2}$, Aimun A. Jamjoom ${ }^{3}$ \\ 1. Neurological Surgery, College of Medicine and Veterinary Medicine, University of Edinburgh, \\ Edinburgh, GBR 2. Neurological Surgery, College of Life Sciences and Medicine, University of Aberdeen, \\ Aberdeen, GBR 3. Neurological Surgery, Centre for Clinical Brain Sciences, University of Edinburgh, \\ Edinburgh, GBR
}

$\square$ Corresponding author: Aimun A. Jamjoom, aabjamjoom@gmail.com Disclosures can be found in Additional Information at the end of the article

\section{Abstract}

Traumatic Brain Injury (TBI) is a growing public health issue with an increasing burden of disease globally. TBI can lead to significant motor, cognitive and emotional deficits. Mobile health (mHealth) is a promising technology to help diagnose and manage patients with TBI. The aim of this study was to systematically examine and classify available TBI mobile applications (apps) and critically appraise the literature underpinning mHealth for the management of TBI. Two major app markets (Apple and Google Play) were systematically searched. Included apps were classified and had data extracted. Coupled to this, a systematic search of the literature (MEDLINE, Web of Science, Scopus, PsycINFO) was performed examining the effectiveness of mHealth interventions in helping patients manage their symptoms after TBI (registered with PROSPERO: CRD42018107386). From 1296 apps, 53 met our inclusion criteria. The top three functions were TBI screening, education and biomechanics monitoring. Twenty-six apps (49.1\%) focused on sports-related concussion. Eight apps (15.1\%) were gamified and 12 apps (22.6\%) connected to an external device. From the literature, a total of eight articles were included of which four (50\%) were case series, two (25\%) were feasibility/pilot studies, one (12.5\%) was a case report, and one (12.5\%) was a randomised controlled trial. The median number of patients was seven $(1-43)$. There is a small number of mobile apps for TBI, mostly focusing on sports-related concussion. At present, the uptake and application of these apps as a management aid is limited and the evidence for their usefulness in TBI remains low.

Received 05/06/2019

Review began 06/03/2019 Review ended 06/28/2019 Published 07/10/2019

\section{(c) Copyright 2019}

Christopher et al. This is an open access article distributed under the terms of the Creative Commons Attribution License CC-BY 3.0., which permits unrestricted use, distribution, and reproduction in any medium, provided the original author and source are credited.
Categories: Neurosurgery, Healthcare Technology, Trauma

Keywords: concussion, mhealth, mobile technology in healthcare, traumatic brain injury (tbi)

\section{Introduction And Background}

Traumatic Brain Injury (TBI) is a growing public health issue with an increasing burden of disease globally [1]. There is a spectrum of TBI severity with severe cases leading to significant motor, cognitive, and emotional deficits. Even mild injuries, which constitute over $80 \%$ of cases, can lead to post-concussional symptoms (this includes a range of symptoms that come under four categories: somatic, cognitive, emotional and sleep related) which can impair patients' function [2]. The challenges in TBI management are numerous including promoting awareness and public education. For concussion or mild TBI, there is a big need for early identification of patients and providing support for post-concussional symptoms. While for more severe TBIs there is a need to deliver effective motor and cognitive rehabilitation. Mobile 
technology provides a potential means to meet a number of these challenges in TBI care. Over recent years, mobile health (mHealth) has emerged as an increasingly promising area for supporting patients and healthcare professionals in a number of diseases such as diabetes and mental health problems $[3,4]$. mHealth refers to the practice of medicine by a mobile device such as a mobile phone, tablet computer and, more recently, wearable sensors. The mobile technology has been used in screening for sports-related concussion and in delivering TBI education [5]. However, despite the promise of mHealth in TBI, there has not been a comprehensive analysis of available mobile applications (apps) and a review of the literature. In this study, we aimed to examine the available commercial mobile apps for TBI and examine the evidence underpinning their use for managing TBI symptoms. To do this, we systematically examined the two largest mobile app markets for apps focused on TBI. Coupled to this, we conducted a systematic review of the literature to understand the underlying evidence for mHealth interventions for helping patients manage their symptoms after a TBI.

\section{Review}

\section{Materials and Methods}

A systematic review of the mobile app market

A systematic review for available mobile apps was performed by searching the mobile app markets Google Play and the Apple App Store between June to December 2017. The mobile app markets were each systematically searched using the following keywords: 'concussion', 'head injury', 'traumatic brain injury' or 'brain injury'. Initial screening was based on the app name and description provided by the developers. Apps were reviewed independently for eligibility by two investigators (EC - Edward Christopher and AJ - Aimun Jamjoom). Apps were eligible for inclusion if they had one of the following functions: TBI education (defined as the provision of written or graphical information about TBI, its diagnosis and its management); TBI symptom management and/or tracking (defined as the functionality permitting users to input data about their symptoms using the app); TBI assessment/screening tool (defined as the functionality permitting users to input data that stratifies/detects/diagnoses TBI or concussion using the app) and TBI biomechanics measurement (defined as the functionality permitting the app to detect, measure and record biomechanical forces related to TBI). Apps that were not in English were excluded from further analyses. Apps that met our inclusion criteria were installed and reviewed independently by two investigators (EC and $\mathrm{AJ}$ ). We extracted a number of data points including: name, availability in Android or iOS devices or both, main function, if the app is sports-focused, connectivity to external device, gamification, user rating, price in pound sterling (£), number of downloads (until February 2018) and Pan European Game Information rating (PEGI) which is an age recommendation (PEGI3; PEGI7; PEGI12; PEGI16 and PEGI18). If cross-platform duplicates were found, only the most recently updated version of the app was included for analyses.

\section{A systematic review of the literature}

We conducted a systematic review with the aim of answering the following question: are mHealth interventions effective in managing patient symptoms after a TBI? The systematic review was registered on the PROSPERO database (CRD42018107386 -

http://www.crd.york.ac.uk/PROSPERO/display_record.php?ID=CRD42018107386) and we used the PRISMA guidelines for reporting of this systematic review [6]. A literature search was performed using MEDLINE (accessed by PubMed), Web of Science, Scopus and PsycINFO in August 2018. A search strategy combining a number of TBI-related phrases was used: (“Traumatic brain injury", "Concussion", "TBI" + "mHealth", "Mobile phone", "Smartphone", "iPhone", "Mobile app") (Table 1). We aimed to include clinical studies that looked at the impact of mHealth in managing patient symptoms after a TBI. We defined mHealth as any mobile 


\section{Cureus}

technology including smartphones or handheld devices, mobile applications, mobile phone text messaging interventions, phone calendar/reminders and sensors linked to mobile devices. Databases were searched from inception to August 2018. The study had to include clinical outcome measures related to TBI symptomology (somatic, emotional, cognitive and sleep). The studies had to focus on TBI patients exclusively and we excluded studies looking at mixed populations of patients with acute brain injuries (such as stroke or post-traumatic stress disorder). We included only English language articles and excluded preclinical studies. Our inclusion criteria of study design included randomised controlled trials (RCT), observational studies (case reports, case series, cohort and case-control studies) and pilot/feasibility studies. For RCTs, we examined the degree of bias using the risk of bias tool found in the Cochrane handbook for systematic reviews of interventions [7]. For case reports or case series, we used a recently proposed framework by Murad and colleagues to examine methodological quality based on four criteria: selection, ascertainment, causality and reporting [8]. Initial screening was based on titles and abstracts which were reviewed by two independent investigators (KA Kareem Alsaffarini and EC). Subsequently, the two investigators independently evaluated fulltext articles and determined eligibility. Any discrepancies were examined by a third investigator (AJ) who made the final decision on the application of inclusion criteria. Included studies had the following data extracted and collated in a data-sheet: author, publication year, type of mHealth Intervention, TBI severity (mild/moderate/severe), number of patients, duration of follow up, outcome measures and key findings. The reference lists of included studies were also reviewed, and any additional eligible studies were included in the review.

\begin{tabular}{|ll}
\hline Code & Search term \\
1 & "Traumatic Brain Injury" \\
2 & "Concussion" \\
3 & "TBI" \\
4 & "Mhealth" \\
5 & "Mobile Phone" \\
6 & "Smartphone" \\
7 & "iPhone" \\
8 & "Mobile App" \\
9 & (1 AND 4) + (1 AND 5) + (1 AND 6) + (1 AND 7) + (1 AND 8) \\
10 & (2 AND 4) + (2 AND 5) + (2 AND 6) + (2 AND 7) + (2 AND 8) \\
11 & (3 AND 4) + (3 AND 5) + (3 AND 6) + (3 AND 7) + (3 AND 8)
\end{tabular}

\section{TABLE 1: Search strategy for systematic review}

Search terms (1-8) were added in pairs and their results combined in search strategy (9-11)

TBI: traumatic brain injury; Mobile app: mobile application 


\section{Cureus}

\section{Results}

A systematic review of the mobile app market

Our search strategy returned 1296 apps of which 563 apps (43.4\%) were intra-platform duplicates. We removed these duplicates and of the remaining 733 apps, 73 apps (10.0\%) met our inclusion criteria. We removed 17 cross-platform duplicates and excluded three apps: two were withdrawn from the platform by the developers during the course of the study and another for being non-English. The final inclusion list consisted of 53 apps (Figure 1). The 53 apps reviewed in this study are outlined in Table 2. Of the apps, 29 (54.7\%) were exclusively available on Google Play, 10 apps (18.9\%) were only available on App Store and 14 apps (26.4\%) were available on both app markets. The majority of apps were updated in the last two years: 2017 (45.3\%) followed by 2016 (26.4\%). We classified apps according to their main function related to TBI. The most common function in 18 of the apps (34.0\%) was as a TBI assessment/screening tool. The three top app functions were: delivery of TBI education (28.3\%), detection of TBI biomechanics forces (20.8\%) and symptom management and/or tracking (17.0\%). Eight apps (15.1\%) were gamified and 12 apps (22.6\%) connected to an external device. 


\section{Cureus}

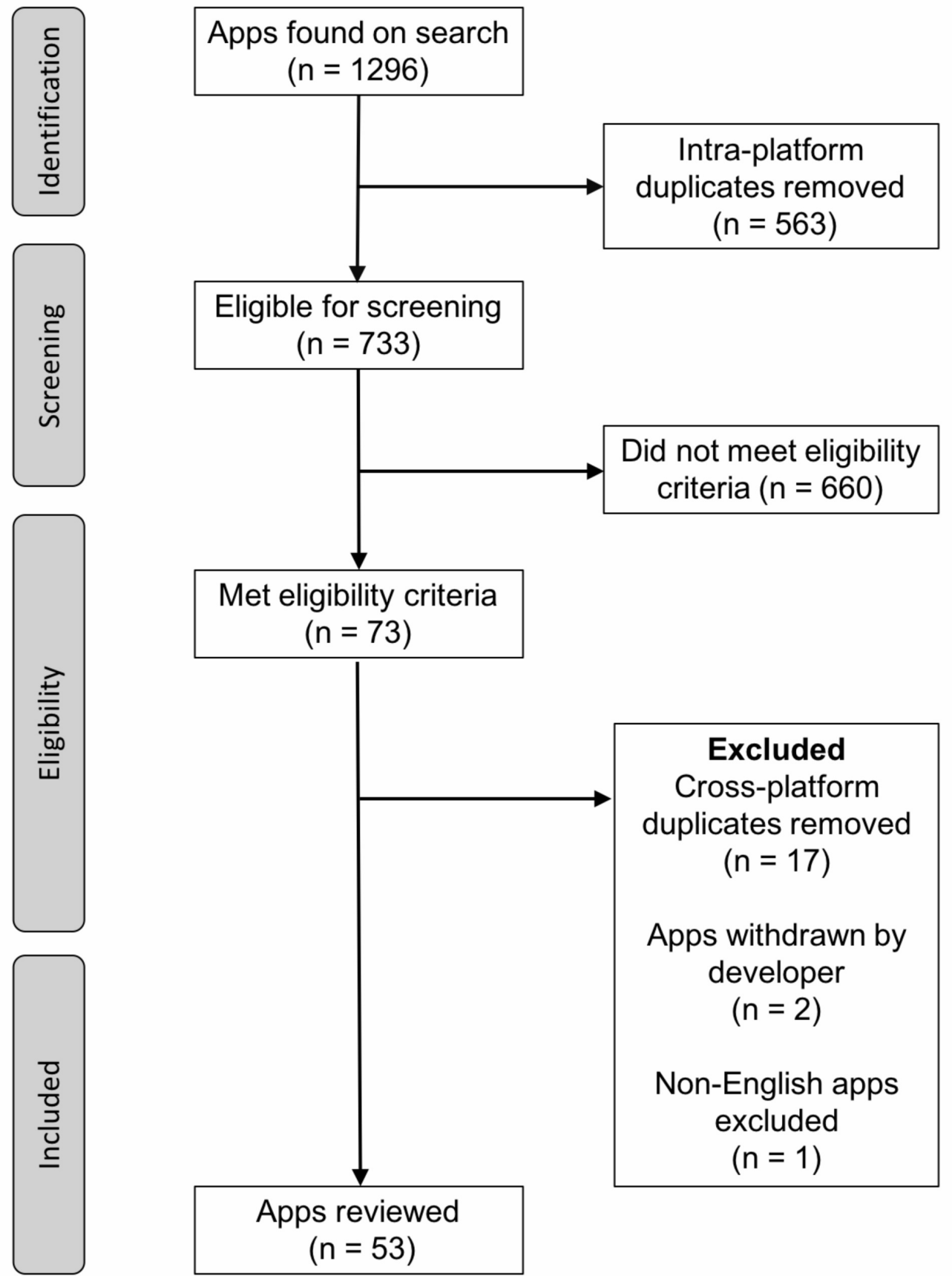

FIGURE 1: Flow chart of included mobile apps in systematic review of mobile app market

Apps: mobile applications

Name

Accident Concussion Scale

\section{Operating} system

External
Gamification

Downloads
Main

function

TBI

assessment/
Price Sports-

(£) focused

iOS 


\section{Cureus}

(ACS)

Al Sport

AoS Concussion Sensor Main

App

Barrow Brainball

Brain Injury

CDC Heads Up Concussion Safety

CDC Heads Up Rocket

Blades - The Brain Safety

Game

CNS Mobile

Concussion Assessment \&

Response

Concussion Awareness

Concussion Buddy

Concussion Coach

Android

Android,

iOS

Android

iOS

iOS

iOS

iOs

Android

Android,

OS

$10-50$

$1-5$

$1000-5000$

$1-5$

assessment/ 4.92 -

screening tool

Symptom

$100-500$

management

\pm tracking

$1000-5000$

TBI education 0

TBI education

5000-10000 TBI education 0

TBI

$500-1000$

assessment/ $3.10+$

screening tool

TBI education $0 \quad+$

Symptom

management 0 -

\pm tracking

Symptom

$500-1000$

management

\pm tracking

TBI

iOS
Android,

iOS

Android

Concussion Management assessment/ 0 + screening tool

Symptom management 0 \pm tracking

$50-100$

TBI education 0

$\begin{array}{llll}- & \begin{array}{l}\text { assessment/ } \\ \text { screening tool }\end{array} & \\ & \begin{array}{l}\text { Symptom } \\ \text { management }\end{array} & 0 & - \\ & \pm \text { tracking } \\ 50-100 & \text { TBl education } & 0 & - \\ 1000-5000 & \text { TBl education } & 0 & -\end{array}$




\section{Cureus}

Concussion Recognition \&
Response
Concussion Signs and
Symptoms
Concussion Test \& Tracker by
SportGait
CSx HeadGuard

Android,

iOS

Android,

iOS

Android

Android

Don't Pull The Plug!

FACT Concussion Test

Android

Android

FirstResponder ${ }^{\mathrm{TM}}$ Concussion App

Android

Head Case

Head Case
Head Injury Association
Headcheck

Android

iOS

iOS

HeadTek
HEADWays

Android +

Android

iOS

HHN Impact Monitor

HitCheck: Sideline

Concussion Testing

Android,

iOS

Android
$10000-$

$1000-5000$

TBI

assessment/ 0 +

screening tool

$1000-5000$

TBI education $\quad 0$

Symptom

$100-500$

management

\pm tracking

TBI

$100-500$

assessment/ $0 \quad+$

screening tool

TBI

$50-100$

assessment/ 0

screening tool

TBI

$100-500$

assessment/

screening tool

TBI

$500-1000$

assessment/ 0 +

screening tool

TBI

50-100 biomechanic 0 +

sensor

TBI education 0

TBI

assessment/ 0

screening tool

TBI

100-500 biomechanic 0 +

sensor

100-500 TBI education 0 -

TBI

biomechanic $0+$ sensor

TBI

assessment/ $0 \quad+$

screening tool

TBI

biomechanic $0+$

sensor

TBI 


\section{Cureus}

\begin{tabular}{|c|c|c|c|c|c|c|c|}
\hline ICEdot & Android & + & - & $1000-5000$ & $\begin{array}{l}\text { biomechanic } \\
\text { sensor }\end{array}$ & 0 & + \\
\hline ImPACT Passport & $\begin{array}{l}\text { Android, } \\
\text { iOS }\end{array}$ & - & - & $5000-10000$ & $\begin{array}{l}\text { Symptom } \\
\text { management } \\
\pm \text { tracking }\end{array}$ & 0 & - \\
\hline InVinci-BULL Shockbox & Android & + & - & $1-5$ & $\begin{array}{l}\text { TBI } \\
\text { biomechanic } \\
\text { sensor }\end{array}$ & 3.13 & + \\
\hline $\begin{array}{l}\text { King-Devick Test with Mayo } \\
\text { Clinic }\end{array}$ & iOS & - & - & - & $\begin{array}{l}\text { TBI } \\
\text { assessment/ } \\
\text { screening tool }\end{array}$ & 0 & + \\
\hline MACE Concussion Evaluation & Android & - & - & $50-100$ & $\begin{array}{l}\text { TBI } \\
\text { assessment/ } \\
\text { screening tool }\end{array}$ & 0 & - \\
\hline Max Impact & $\begin{array}{l}\text { Android, } \\
\text { iOS }\end{array}$ & - & + & $10-50$ & $\begin{array}{l}\text { Symptom } \\
\text { management } \\
\pm \text { tracking }\end{array}$ & 0 & - \\
\hline Medrills: Skull and Brain & $\begin{array}{l}\text { Android, } \\
\text { iOS }\end{array}$ & - & - & $10-50$ & TBI education & 3.13 & - \\
\hline mTBI Pocket Guide & Android & - & - & $\begin{array}{l}10000- \\
50000\end{array}$ & TBI education & 0 & - \\
\hline PlayerMD Biometric Plattorm & Androld & + & - & $100-500$ & $\begin{array}{l}\text { TBI } \\
\text { biomechanic } \\
\text { sensor }\end{array}$ & 0 & + \\
\hline Pocket TBI & Android & - & - & $5000-1000$ & TBI education & 0 & - \\
\hline Rancho Los Amigos Scale & Android & - & - & 5000-10000 & $\begin{array}{l}\mathrm{TBI} \\
\text { assessment/ } \\
\text { screening tool }\end{array}$ & 0 & - \\
\hline $\begin{array}{l}\text { Rebound: Beating } \\
\text { Concussions }\end{array}$ & $\begin{array}{l}\text { Android, } \\
\text { iOS }\end{array}$ & - & + & $10-50$ & TBI education & 0 & + \\
\hline Return2Play for Concussion & Android & - & - & $500-1000$ & $\begin{array}{l}\text { Symptom } \\
\text { management } \\
\pm \text { tracking }\end{array}$ & 0 & + \\
\hline SACTool Beta & Android & - & - & $500-1000$ & $\begin{array}{l}\text { TBI } \\
\text { assessment/ } \\
\text { screening tool }\end{array}$ & 0 & - \\
\hline Shockbox & Android & + & - & $1000-5000$ & $\begin{array}{l}\text { TBI } \\
\text { biomechanic } \\
\text { sensor }\end{array}$ & 0 & + \\
\hline SwiftReact & Android & + & + & $10-50$ & $\begin{array}{l}\text { Symptom } \\
\text { management } \\
\pm \text { tracking }\end{array}$ & 0 & - \\
\hline
\end{tabular}




\section{Cureus}

\begin{tabular}{|c|c|c|c|c|c|c|c|}
\hline TBI Prognosis Calculator & $\begin{array}{l}\text { Android, } \\
\text { iOS }\end{array}$ & - & - & $1000-5000$ & $\begin{array}{l}\text { TBI } \\
\text { assessment/ } \\
\text { screening tool }\end{array}$ & 0 & - \\
\hline The Blast Gauge System App & Android & + & - & $10-50$ & $\begin{array}{l}\text { TBI } \\
\text { biomechanic } \\
\text { sensor }\end{array}$ & 0 & - \\
\hline World Rugby Concussion & $\begin{array}{l}\text { Android, } \\
\text { iOS }\end{array}$ & - & - & $1000-5000$ & TBI education & 0 & + \\
\hline X2 ICE & iOs & - & - & - & $\begin{array}{l}\text { TBI } \\
\text { assessment/ } \\
\text { screening tool }\end{array}$ & 0 & + \\
\hline XLNTbrain-mobile & Android & - & - & $500-1000$ & $\begin{array}{l}\text { TBI } \\
\text { assessment/ } \\
\text { screening tool }\end{array}$ & 0 & + \\
\hline
\end{tabular}

\section{TABLE 2: Summary of included mobile app characteristics}

App: mobile application; TBI: traumatic brain injury; CDC: Centers for Disease Control; CNS: central nervous system

In terms of the target audience, nine apps (17.0\%) were designated for healthcare professionals, 18 apps (34.0\%) were designated for patients and 26 apps (49.1\%) were designated for athletes. The majority of apps (66.0\%) were rated PEGI3, followed by PEGI7 (13.2\%), PEGI12 (5.7\%), and PEGI18 (1.9\%). Seven apps (13.2\%) were unrated. In terms of user rating, the median rating was 4.2 stars out of five [Interquartile range (IQR) 3.5 - 5, n=35] with the median number of ratings per app being five (IQR 2 - 13, $n=35$ ). We excluded 18 apps from these analyses owing to them having no user rating at all. Price ranged from free to $£ 4.92$ but most apps (92.5\%) were free. Google Play provides the number of downloads for each app in ranges. Taking the middle of the range as the number of downloads for each app, the median number of downloads was 300 (IQR 75 - 3000, n=43) for apps available on Google Play. The Apple App Store does not provide download metrics and thus we were unable to analyse these data for apps exclusively available on App Store $(\mathrm{n}=10)$.

\section{Sports-focused apps}

We examined in detail the 26 apps that were designated for athletes since they formed the majority in our final inclusion list. Fifteen apps (57.7\%) were exclusively available on Google Play, five apps (19.2\%) were exclusively available on App Store and six apps (23.1\%) were available on both. We classified these sports-focused apps according to their main function: 10 apps (38.5\%) focused on TBI assessment/screening tool, four apps (15.4\%) delivered sportsrelated TBI education and two apps (7.7\%) focused on symptom management \pm tracking. Four apps (15.4\%) were gamified. Ten apps (38.5\%) could accommodate connectivity to an external device. Of these, six apps could accommodate only one device, three apps could accommodate two devices, and one app could accommodate up to four external devices. External devices included mouth guards, impact sensors, headbands, accelerometers, gyroscopes, thermometers, helmets, and skullcaps. In terms of user rating, the median rating was 4.2 stars out of five (IQR 3.9 - 5, n=17) with the median number of ratings per app being five (IQR 2 - 13, $\mathrm{n}=17$ ); we excluded nine apps from these analyses owing to them having no user rating. Price 
ranged from free to $£ 3.13$ but most sports-focused apps (92.3\%) were free. Google Play provides the number of downloads for each app in ranges. Taking the middle of the range as the number of downloads for each app, the median number of downloads was 300 (IQR $75-3000$, n=21) for apps available on Google Play. App Store does not provide download metrics and thus we were unable to analyse these data for apps exclusively available on App Store $(n=5)$.

\section{A systematic review of the literature}

A total of 181 articles returned using our search strategy in August 2018. After removing duplicates, a total of 116 papers remained. The abstract screening was performed, and 29 papers were chosen for a more detailed review of the full article. Seven articles met our final inclusion criteria from which one further article was added after an examination of the reference lists (Figure 2). A summary of the eight included studies can be found in Table 3 [815]. Of the included studies, four (50\%) were case series, two (25\%) were feasibility/pilot studies, one (12.5\%) was a case report, and one (12.5\%) was an RCT [14]. We examined the risk of bias in the RCT using the Cochrane Risk of Bias Tool: random sequence generation (low risk), allocation concealment (high risk), blinding of participants and personnel (high risk), blinding of outcome assessment (high risk), incomplete outcome data (low risk) and selective reporting (low risk). Based on the Murad criteria, we found that one of the case reports was high quality while the rest were of medium quality. The median number of patients was seven (1 - 43). The most common investigation in five (62.5\%) articles was the role of mHealth intervention as a memory aid (including goal setting) after TBI. The remaining three (37.5\%) studies looked at mHealth intervention as a tool for symptom/mood management after TBI. Seven studies (87.5\%) examined a mobile app as the mHealth intervention in TBI. 


\section{Cureus}

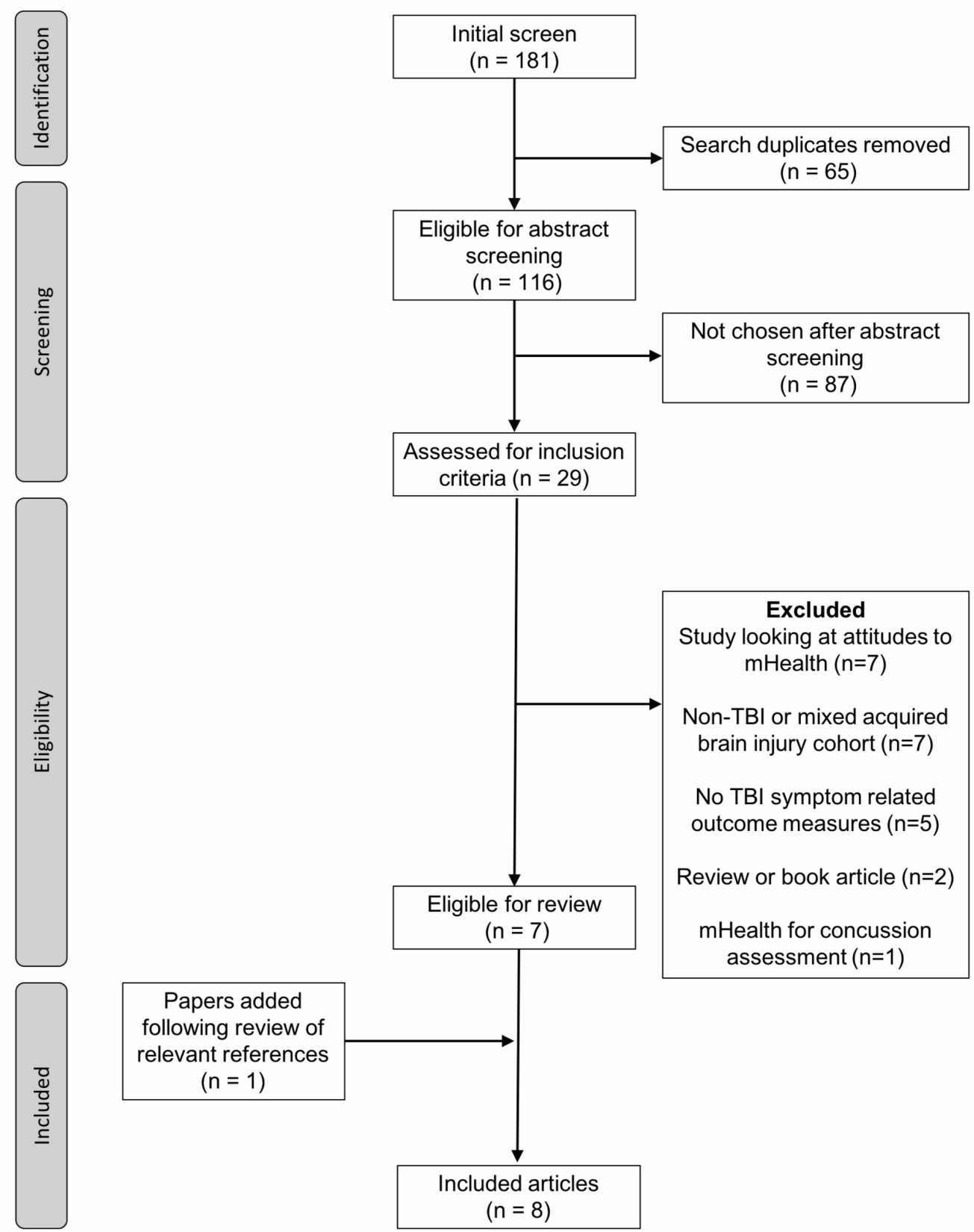

\section{FIGURE 2: Flow chart of included studies in systematic review}

of literature

TBI: traumatic brain injury

\begin{tabular}{|c|c|c|c|c|c|c|c|c|}
\hline Author & $\begin{array}{l}\text { mHealth } \\
\text { Intervention }\end{array}$ & Study Type & $\begin{array}{l}\text { TBI } \\
\text { Severity }\end{array}$ & $\begin{array}{l}\text { Number of } \\
\text { Patients }\end{array}$ & Outcome Measures & $\begin{array}{l}\text { Duration of } \\
\text { follow-up }\end{array}$ & Quality & Key Findings \\
\hline & Google & & & & $\begin{array}{l}\% \text { target events forgotten; } \\
\text { The Beliefs about Memory }\end{array}$ & $\begin{array}{l}\text { Six weeks of } \\
\text { baseline data }\end{array}$ & & $\begin{array}{l}\text { Objective } \\
\text { improvement in } \\
\text { target events } \\
\text { following } \\
\text { intervention; }\end{array}$ \\
\hline
\end{tabular}




\section{Cureus}

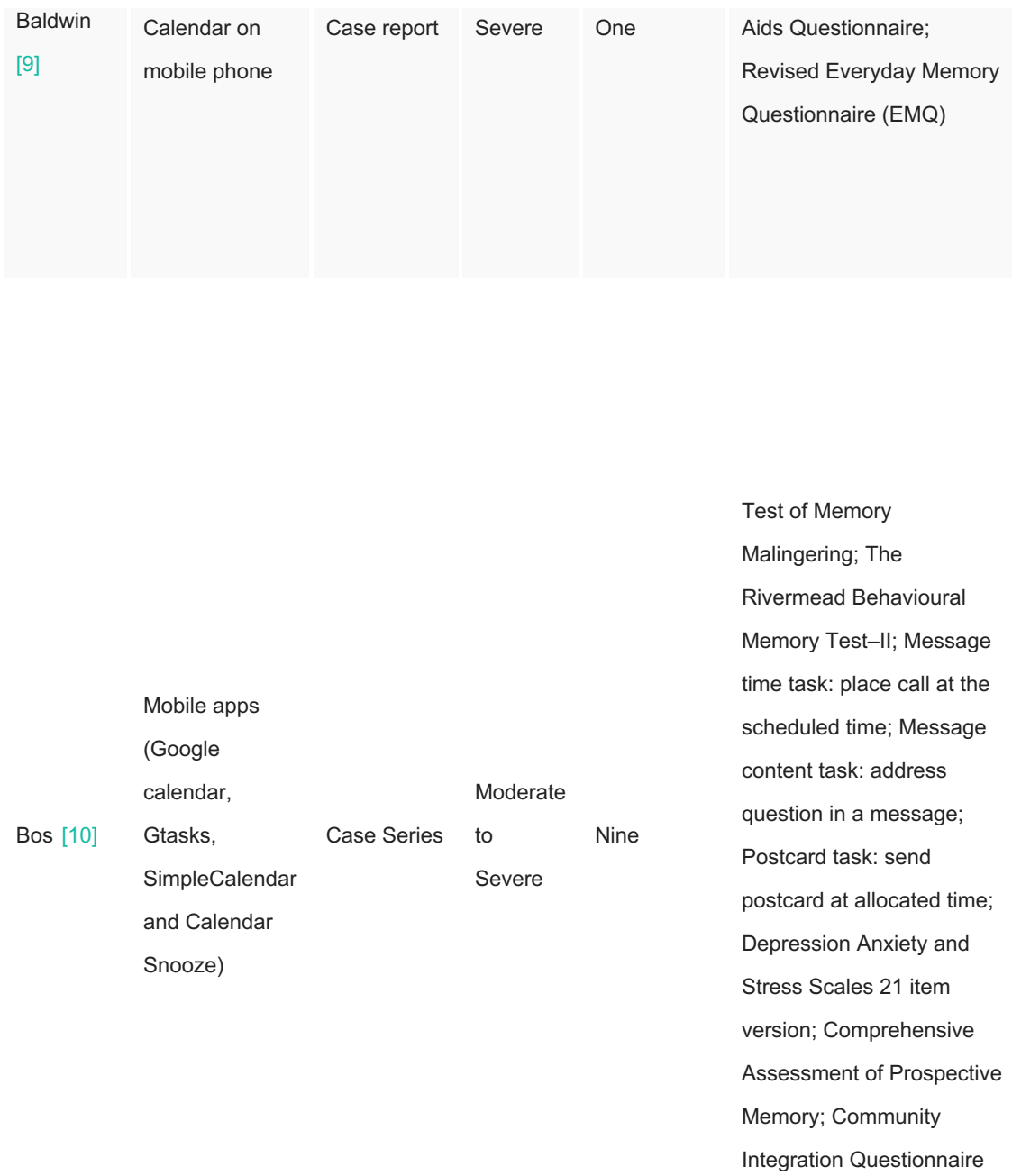

and six weeks High
of intervention
data
data

Eight weeks Medium

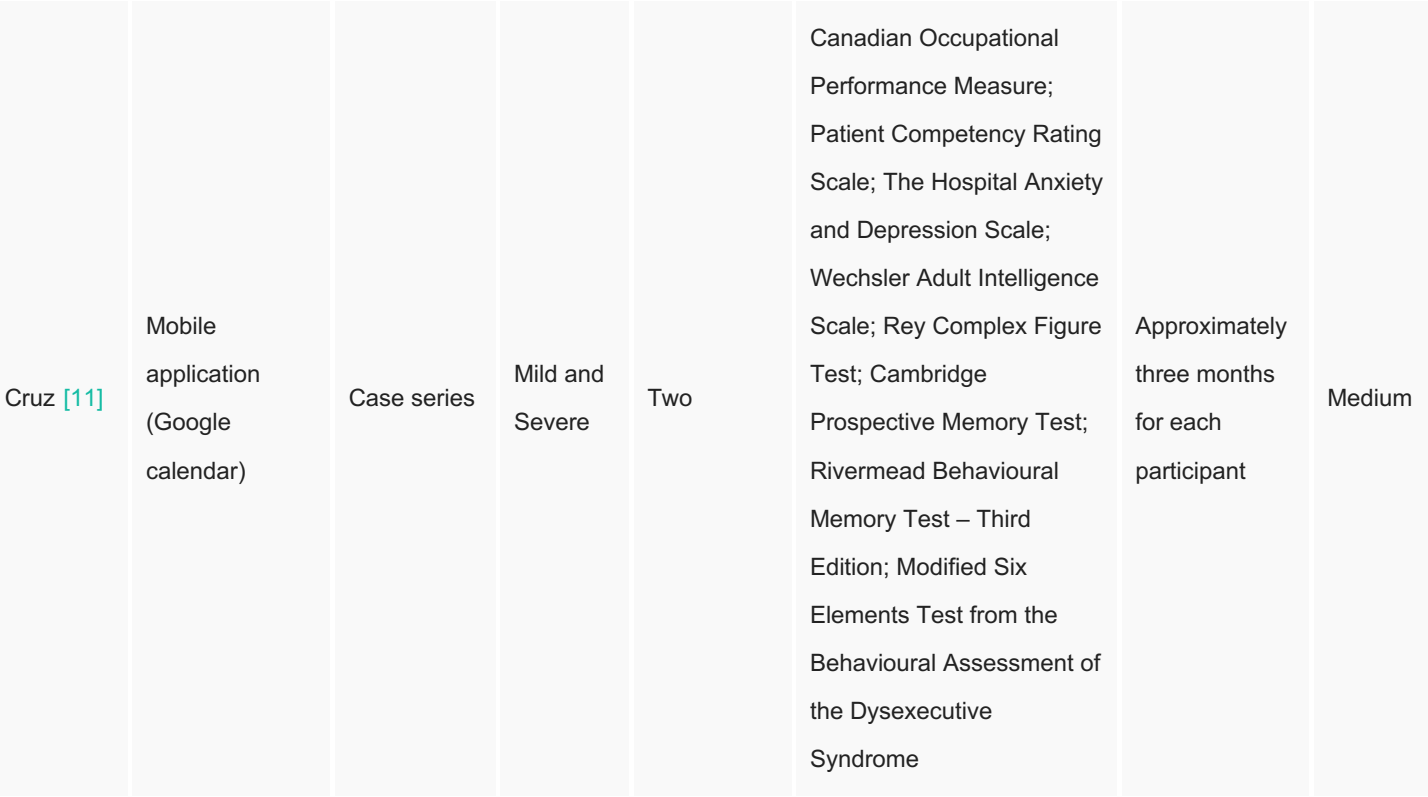

Mobile text reminders

led to an

improvement in

performance and

satisfaction;

Reminders had to be tailored to the

individual to

maximise impact:

The use of different

reminder modalities

helps improve the

effectiveness

Participants correctly

completed $73.4 \%$ of 


\section{Cureus}

$\begin{array}{llll} & \text { Tracking mood- } & & \\ & \text { related } & & \\ & \text { symptoms } & & \\ \text { Juengst } & \text { using a } & \text { Feasibility } & \text { Mild to } \\ {[12]} & \text { smartphone } & \text { Study } & \text { Severe } \\ & \text { application } & & \\ & \text { (iPerform } & \\ & \text { platform) } & & \\ & & \end{array}$

App-based

coaching

Narad

[13] intervention

(SPAN) for

adolescents

with TBI
Compliance measured the

total number of completed

assessments divided the

total number of scheduled

assessments; Satisfaction

measured with 7-point

Likert scale assessing

usability and satisfaction

with the application:

Telehealth Usability

Questionnaire (TUQ) all scheduled

assessments;

Participants reported

high satisfaction with

smartphone

applications and

found them easy to

use; Comparison of

Eight weeks Medium

assessments

obtained via

telephone-based

interview and EMA

demonstrated high

correlations;

Participants reported

high satisfaction

across the eight

weeks of using the

iPerform application

All participants felt

app provided

appropriate

scaffolding for goal

setting and

management; Two of

the four participants

completed all steps,

achieved their goals

and identified

additional goals to

work after completion

of the SPAN

program; No

statistically significant differences between pre- and post-

intervention scores

for social

competency and

social problems

For two participants,

there was an

increase in the target

behaviours achieved

when the phone was

used; The

percentage of target

behaviours achieved

did not return to

initial baseline levels of different

baseline data

(2, 3 and 4

weeks) seven
Using a

'reminders'
Rivermead Behavioural

Memory Test; The map 


\section{Cureus}

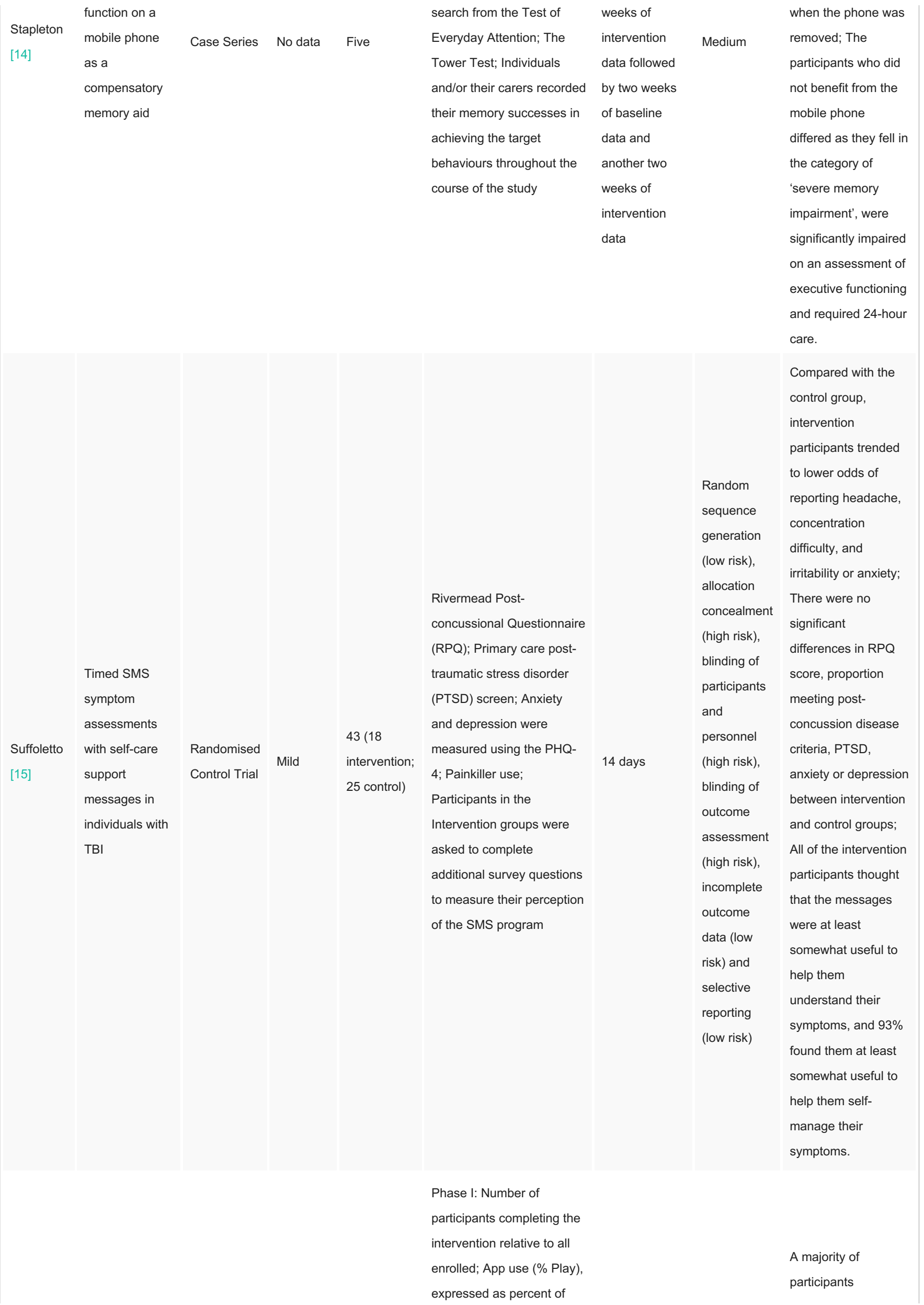




\section{Cureus}

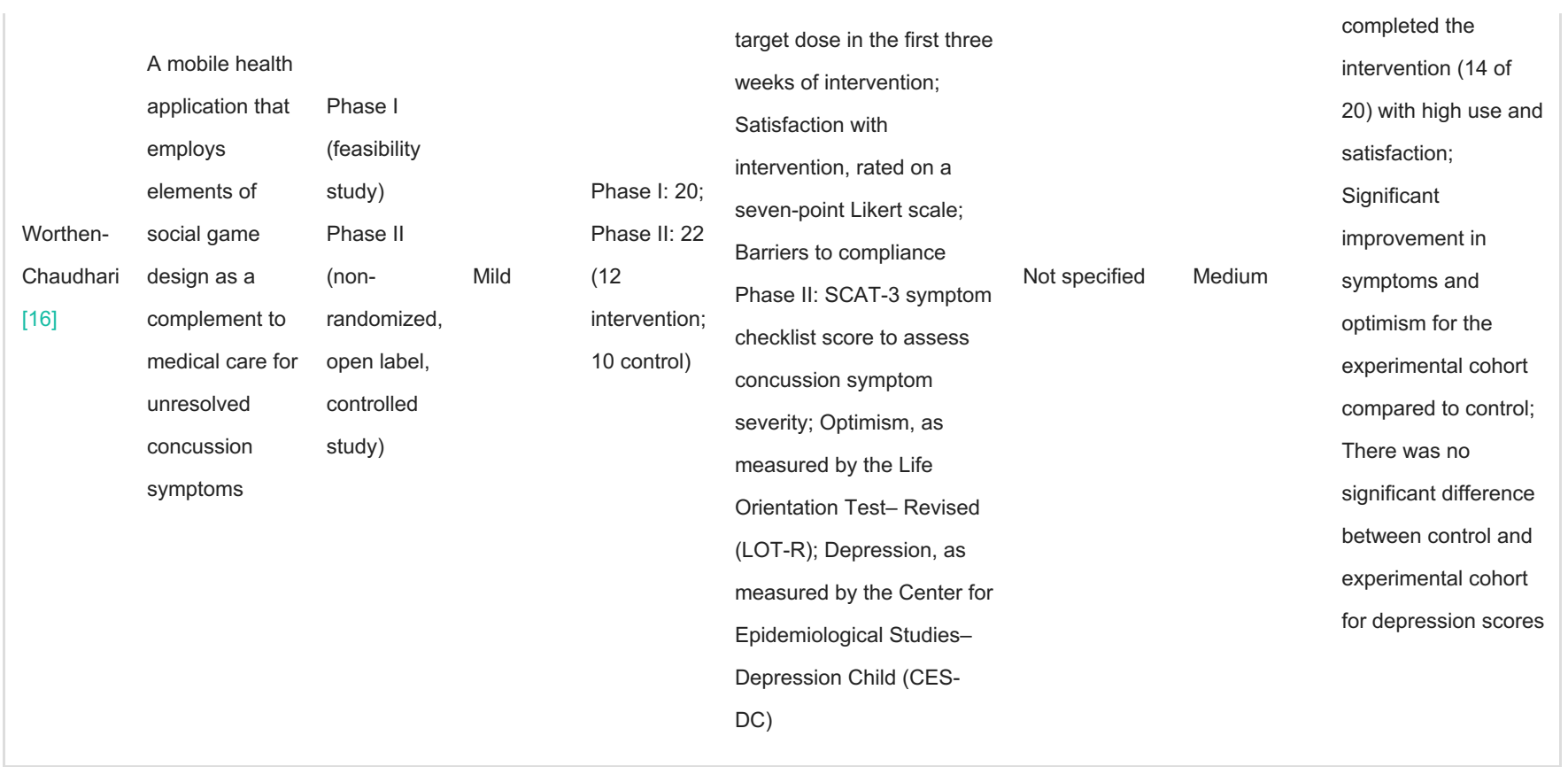

\section{TABLE 3: Summary of included mHealth in TBI studies}

mHealth: mobile health; EMA: ecological momentary assessment; SPAN: Social Participation and Navigation; PHQ-4: patient health questionnaire 4; EMQ: Everyday Memory Questionnaire; TUQ: Telehealth Usability Questionnaire; YSR: Youth Self Report; CBCL: Child Behavior Checklist; RPQ: Rivermead Post-concussional Questionnaire; PTSD: Post-traumatic stress disorder; LOT-R: Life Orientation Test- Revised; CES-DC: Center for Epidemiological Studies-Depression Child

\section{Discussion}

mHealth is increasingly used in medical practice and has the potential to improve healthcare delivery through enhanced communication, data capture, patient monitoring, education and the delivery of digital interventions at scale [17]. A systematic review looking at the role in mHealth for rehabilitation identified seven studies that focused on the use of mHealth for TBI rehabilitation [18]. The study showed that mHealth interventions have been investigated for TBI rehabilitation in a range of areas including reminder support, managing mood-related symptoms and cognitive rehabilitation. TBI is a major cause of injury-related death and disability globally [1]. However, the spectrum of the disease from mild to severe means different patient cohorts require different support strategies. All TBI patients require support with understanding and managing their symptoms such as headaches, mood disturbance and difficulty sleeping. However, patients with more severe injuries with cognitive and motor deficits require tools to support them such as reminder notifications, cognitive and motor rehabilitation.

Mobile devices are becoming increasingly accessible and they have the potential to play a central part in TBI education, screening, and management. Our systematic review demonstrated that a relatively small number of TBI apps are currently commercially available. This is considerably smaller in comparison to other pathologies such as diabetes and depression which were found to have over 1000 apps [19]. This difference is disproportionately large considering the global burden of TBI and particularly as a recent Lancet Neurology Commission highlighted the suboptimal state of TBI diagnosis, monitoring, and management in parts of the world; areas where mHealth could provide solutions [20].

Of the included mobile apps, we found a low level of user engagement with a median of 300 
downloads and only an upper quartile of 3000. Given the epidemiology of TBI and its growing public profile, this shows very limited uptake of these mobile apps among patients and healthcare professionals. Wong and colleagues looked at the attitudes of patients with TBI towards the use of a mobile phone and found positive views regarding the benefits of mobile phones as a means of communication and memory aid [21]. It does suggest however that the current commercial TBI app market landscape is not being actively used by the target audience. This may be because TBI patients' needs are being met by other more established programmes such as reminder/calendar apps native to the patients' mobile phones. Coupled to this, medical apps are not tightly regulated and users may question their accuracy. This was highlighted by a recent systematic review which looked at the compliance of concussion assessment apps and found a wide range of compliance rates with a concussion assessment tool [5]. This is concerning, given that the most common app function in our review was TBI screening/assessment. There are growing efforts to provide organised peer review of apps in platforms such as TopOrthoApps and iMedicalApps. The National Health Service (NHS) is currently running the beta site Digital Apps Library which lists apps which meet the NHS quality standards for clinical effectiveness, safety, usability and accessibility and has evidence to support its use.

In the second stage of this study, we performed a systematic review of the literature to look for the evidence underpinning the use of mHealth in managing patient symptoms after TBI. We found only eight studies of which five were either case reports or case series reflecting a very limited evidence base. These studies provide anecdotal evidence of the benefits of mobile apps for patients with TBI. A number of these reports used baseline control data and compared it to mHealth intervention phases which showed varying degrees of improvement in task achievement and satisfaction. Of the controlled studies, Worthen-Chaudhari et al. looked at a gamified mobile app as a tool to help improve post-concussional symptoms in adolescents [15]. In this feasibility study, the investigators found the majority of participants completed the intervention and that there was an improvement in symptom burden in the intervention group. This contrasted to the only RCT in the included studies which looked at a short messaging service (SMS) based intervention for patients following concussion [15]. The investigators found that an intervention of text message-based advice on symptom control did not lead to a significant reduction in symptom burden. Collectively, this review has shown a wide range of mHealth interventions have been tested for patients with TBI. The anecdotal evidence shows potential for mHealth interventions to help individuals with TBI. However, the evidence base is limited, and we conclude that there is currently insufficient data to support the use of mHealth in managing symptoms after TBI.

Our study has a number of limitations. We only looked at iOS and Android markets which may mean we missed some mobile apps for other platforms such as Microsoft or Blackberry. Also, we only examined studies focusing on managing TBI patients and excluded other studies that look at different aspects of mHealth in TBI care. Despite these drawbacks, we believe that the data presented in this study is the most comprehensive examination of mHealth in TBI to date. It demonstrates a limited number of commercially available mobile apps that have little uptake from patients and healthcare professionals based on the download numbers. Coupled to this, we found that the evidence base underpinning mHealth interventions in TBI to be mainly anecdotal in the form of case reports and case series.

\section{Conclusions}

Despite a growth in the use of mHealth for other diseases, our study found only a small number of mobile apps for TBI with limited uptake based on download metrics. The majority of these focused on sports-related concussion and were aimed at either concussion screening or TBI education. Coupled to this, the evidence underpinning mHealth in TBI is limited with a dearth of large clinical trials examining this area. Given the range of care needs TBI patients have, 
there is scope to develop TBI-specific mHealth interventions that aim to help patients that are robustly tested for clinical effectiveness.

\section{Additional Information \\ Disclosures}

Conflicts of interest: In compliance with the ICMJE uniform disclosure form, all authors declare the following: Payment/services info: All authors have declared that no financial support was received from any organization for the submitted work. Financial relationships: All authors have declared that they have no financial relationships at present or within the previous three years with any organizations that might have an interest in the submitted work. Intellectual property info: $\mathrm{AABJ}$ is developing a prototype for a web application to support patients with concussion. This work has been funded by the National Institute of Health Research. No patent is currently planned though may be in future. . Other relationships: All authors have declared that there are no other relationships or activities that could appear to have influenced the submitted work.

\section{Acknowledgements}

AABJ is supported by the National Institute for Health Research (NIHR) Brain Injury MedTech Co-operative based at Cambridge University Hospitals NHS Foundation Trust and University of Cambridge. The views expressed are those of the author(s) and not necessarily those of the NHS, the NIHR or the Department of Health.

\section{References}

1. Roozenbeek BR, Maas AI, Menon DK: Changing patterns in the epidemiology of traumatic brain injury. Nat Rev Neurology. 2013, 9:231-236.

2. Hou R, Moss-Morris R, Peveler R, Mogg K, Bradley BP, Belli A: When a minor head injury results in enduring symptoms: a prospective investigation of risk factors for postconcussional syndrome after mild traumatic brain injury. J Neurol Neurosurg Psychiatry. 2012, 15:217-223. 10.1136/jnnp-2011-300767

3. Kitsiou S, Paré G, Jaana M, Gerber B: Effectiveness of mHealth interventions for patients with diabetes: An overview of systematic reviews. PLoS One. 2017, 23:1-16.

10.1371/journal.pone.0173160

4. Shen N, Levitan MJ, Johnson A, Bender JL, Hamilton-Page M, Jadad AA, Wiljer D: Finding a depression app: a review and content analysis of the depression app marketplace. JMIR mHealth uHealth. 2015, 3:e16. 10.2196/mhealth.3713

5. Lee H, Sullivan SJ, Schneiders AG, et al.: Smartphone and tablet apps for concussion road warriors (team clinicians): a systematic review for practical users. Br J Sports Med. 2015, 49:499-505. 10.1136/bjsports-2013-092930

6. Liberati A, Altman DG, Tetzlaff J, et al.: The PRISMA statement for reporting systematic reviews and meta-analyses of studies that evaluate healthcare interventions: explanation and elaboration. BMJ. 2009, 339:b2700. 10.1136/bmj.b2700

7. Higgins JP, Green S: Cochrane handbook for systematic reviews of interventions. The Cochrane Collaboration, 2009.

8. Murad MH, Sultan S, Haffar S, Bazerbachi F: Methodological quality and synthesis of case series and case reports. BMJ Evid Based Med. 2018, 23:60-63. 10.1136/bmjebm-2017-110853

9. Baldwin VN, Powell T: Google Calendar: A single case experimental design study of a man with severe memory problems. Neuropsychol Rehabil. 2015, 25:617-636.

10.1080/09602011.2014.956764

10. Bos HR, Babbage DR, Leathem JM: Efficacy of memory aids after traumatic brain injury: A single case series. NeuroRehabilitation. 2017, 41:463-481. 10.3233/NRE-151528

11. Cruz G, Petrie S, Goudie N, Kersel D, Evans J: Text messages reduce memory failures in adults with brain injury: A single-case experimental design. Br J Occup Ther. 2016, 79:598-606.

10.1177/0308022616640299 
12. Juengst SB, Graham KM, Pulantara IW, et al.: Pilot feasibility of an mHealth system for conducting ecological momentary assessment of mood-related symptoms following traumatic brain injury. Brain Inj. 2015, 29:1351-1361. 10.3109/02699052.2015.1045031

13. Narad ME, Bedell G, King JA, Johnson J, Turkstra LS, Haarbauer-Krupa J, Wade SL: Social Participation and Navigation (SPAN): Description and usability of app-based coaching intervention for adolescents with TBI. Dev Neurorehabil. 2018, 21:439-448.

10.1080/17518423.2017.1354092

14. Stapleton S, Adams M, Atterton L: A mobile phone as a memory aid for individuals with traumatic brain injury: a preliminary investigation. Brain Inj. 2007, 21:401-411.

10.1080/02699050701252030

15. Suffoletto B, Wagner AK, Arenth PM, Calabria J, Kingsley E, Kristan J, Callaway CW: Mobile phone text messaging to assess symptoms after mild traumatic brain injury and provide selfcare support: a pilot study. J Head Trauma Rehabil. 2013, 28:302-312.

10.1097/HTR.0b013e3182847468

16. Worthen-Chaudhari L, McGonigal J, Logan K, Bockbrader MA, Yeates KO, Mysiw WJ: Reducing concussion symptoms among teenage youth: Evaluation of a mobile health app . Brain Inj. 2017, 31:1279-1286. 10.1080/02699052.2017.1332388

17. Steinhubl SR, Muse ED, Topol EJ: The emerging field of mobile health. Sci Transl Med. 2015, 7:283rv3. 10.1126/scitranslmed.aaa3487

18. Nussbaum R, Kelly C, Quinby E, Mac A, Parmanto B, Dicianno BE: Systematic Review of Mobile Health Applications in Rehabilitation. Arch Phys Med Rehabil. 2019, 100:115-127. 10.1016/j.apmr.2018.07.439

19. Martínez-Pérez B, de la Torre-Díez I, López-Coronado M: Mobile health applications for the most prevalent conditions by the World Health Organization: review and analysis. J Med Internet Res. 2013, 15:e120. 10.2196/jmir.2600

20. Johnson WD, Griswold DP: Traumatic brain injury: a global challenge . Lancet Neurol. 2017, 16:949-950. 10.1016/S1474-4422(17)30362-9

21. Wong D, Sinclair K, Seabrook E, McKay A, Ponsford J: Smartphones as assistive technology following traumatic brain injury: a preliminary study of what helps and what hinders. Disabil Rehabil. 2017, 39:2387-2394. 10.1080/09638288.2016.1226434 